\title{
DISTRIBUIÇÃO DA ASSIMILAÇÃO DE NITRATO E DE MATÉRIA SECA EM PLANTAS JOVENS DE CAFÉ CULTIVADAS EM DIFERENTES NÍVEIS DE NITROGÊNIO (')
}

\author{
MARIA LUIZA CARVALHO CARELLI $(2,3) \bullet$ JOEL IRINEU FAHL $(2,3)$
}

\begin{abstract}
RESUMO
Fol estudado o efeito de nívels de nitrogênio na distribuiçäo do processo de redução de nitrato e de matéria seca entre as raizes e a parte aérea, em plantas de café de nove meses de idade, cultivadas em areia em casa de vegetaçäo e irrigadas com soluçōes nutritivas contendo $3,75,7,5$ e $15 \mathrm{mM}$ de nitrato. A área foliar e a matéria seca das folhas aumentaram com o nível de nitrato das soluçōes, enquanto a matéria seca das raizes e o número de pares de folhas no ramo ortotrópico nảo foram alterados. A atividade in vivo da redutase de nitrato nas raizes mais jovens fol bem maior do que a das folhas recém-expandidas e aumentou com a concentração de nitrato no meio de crescimento. Os valores da atividade enzimática, quando determinada com a adição de nitrato no meio de reação, foram superiores às análises sem nitrato, sugerindo que a atividade da redutase de nitrato nas ráżes $\theta$, principalmente, nas folhas, fol limitada pela quantidade de substrato. $O$ aumento na disponibilidade de nitrogênio para as plantas proporcionou major crescimento da parte aérea, em relaçāo às raizes.
\end{abstract}

Termos de Indexaçio: caft, crescimento da planta, redutase de nitrato, níveis do nitrogênio.

\section{ABSTRACT}

\section{DISTRIBUTION OF NITRATE ASSIMILATION AND DRY MATTER IN YOUNG COFFEE PLANTS CULTIVATED IN DIFFERENT LEVELS OF NITROGEN}

Nitrate assimilation and plant growth were examined in young coffee plants cultivated in sand in the greenhouse. The plants were supplied with nutrient solutions containing 3.75, 7.5and $15 \mathrm{mM}$ of nitrate. The distribution among the roots and shoots was assessed. Leaf area and leaf dry woight increased with nitrate concentration, while root dry weight and leaf pair number remained constant. The in vivo nitrate reductase activity, assayed with and without exogenous nitrate in root tips was higher than in recently expanded leaves, and increased with nitrate concentration. The highest nitrate reductase activity, assayed with exogenous nitrate, revealed limitation to nitrate assimilation by substrate avallability in both leaves and roots. Shoot growth was higher than root growth as a function of increasing nitrogen levels.

Index terms: coffee, plant growth, nitrate reductase, nitrogen lovels.

(') Recobido para publicaçāo em 6 de agosto de 1990 e acelto em 4 de fovereiro de 1991.

(7) Segăo de Fisiologia, Instituto Agronómico (IAC), Caixa Postal 28, 13001 Campinas (SP).

(9) Com bolsa de pesquisa do CNPq.ABSTRACT 


\section{INTRODUÇĀO}

O nitrogênio absorvido pelas plantas, em sua grande maioria na forma de nitrato, pode ser assimilado nas raízes ou na parte aérea. A parcela com que cada um desses sistemas contribui para a assimilaçăo de nitrogênio pela planta varia grandemente com a espécie e com as condiçóes ambientes. A maioria das espécies herbáceas, incluindo importantes culturas econômicas, reduzem nitrato tanto nas raízes como nas folhas. Em culturas como o algodăo (RADIN, 1977), cevada (CHANTAROTWONG et al., 1976; ASLAM \& HUFFAKER, 1982; LEWIS et al., 1982), soja (CRAFTS-BRANDNER \& HARPER, 1982) e milho (HAGEMAN \& FLESHER, 1960; WALLACE, 1973), os níveis de redutase de nitrato sāo mais altos na parte aérea do que nas raízes. Em outras espécies, como girassol WEISSMAN, 1972), tremoço e rabanete (PATE, 1980), as raízes apresentam atividade bem maior do que a das folhas.

No cafeeiro, tanto as raizes como as folhas assimilam nitrato; nas raizes, os níveis de atividade da redutase de nitrato são, em alguns casos, bem maiores do que os das folhas, variando, entretanto, com a idade da planta e do tecido analisado e, provavelmente, com as condiçóes de crescimento (QUEIROZ et al., 1985, CARELLI, 1987).

A assimilação de nitrato requer energia e esqueletos de carbono que, em órgāos heterotróficos como as raízes, são fornecidos pela oxidaçăo dos carboidratos translocados da parte aérea. O nitrato é um poderoso competidor pela energia disponivel nas raizes, de tal forma que, em plantas cultivadas em alto nivel de nitrato, o teor de açúcares e o crescimento do sistema radicular sāo bem inferiores aos daqueles crescidos em baixo teor de nitrato, ou outras formas de nitrogênio (RADIN et al., 1978; ASLAM \& HUFFAKER, 1982, SUTHERLAND et al., 1985). Tal situaçäo pode ser ainda mais acentuada em plantas como o café, que reduzem grande parte do nitrato absorvido em seu sistema radicular, desde que o custo energético para a assimilação de nitrato nas raízes é quase o dobro daquele observado em tecidos verdes na luz (SCHRADER \& THOMAS, 1981). Isso ocorre porque nas raízes toda a energia necessária para converter nitrato em aminoácidos deriva da oxidaçăo dos carboidratos ou ácidos orgânicos, enquanto, nas folhas, parte desse processo está acoplado diretamente com as reaçōes da luz que ocorrem nos cloroplastos (SCHRADER \& THOMAS, 1981).

$O$ objetivo do presente trabalho foi avaliar os efeitos da concentraçăo de nitrato no meio externo sobre o crescimento e a distribuição do processo de assimilação de nitrato entre as raízes e a parte aérea de plantas de café.

\section{MATERIAL E MÉTODOS}

Foram utilizadas sementes de café (Coffea arabica L.), cultivar Catuaí VermelhoH2077-2-5-81, fornecidas pela Seção de Genética dolnstituto Agronômico. 
As plantas foram obtidas em casa de vegetaçăo, pelo plantio direto de sementes sem pergaminho, em sacos de polietileno com capacidade para dois litros e contendo areia lavada. Até a emergência, as plantas foram irrigadas com água e, a partir do estádio de abertura das folhas cotiledonares ("orelha-de-onça"), passaram a receber o mesmo volume, em dias alternados, de água ou de soluçōes nutritivas com 3,75, 7,5 e 15mM de nitrato. A quantidade de água ou de soluçāo com que as plantas foram irrigadas foi calculada de modo a saturar os vasos até livre percolagem. A solução nutritiva $15 \mathrm{mM}$ de nitrato corresponde a solução completa $n^{2} 1$ de HOAGLAND \& ARNON (1939), e as concentraçöes de 3,75 e 7,5mM foram obtidas, substituindo-se parte do $\mathrm{Ca}\left(\mathrm{NO}_{3}\right)_{2}$ edo $\mathrm{KNO}_{3}$ por $\mathrm{CaCl}_{2}$ e $\mathrm{KCl}$ respectivamente, conforme descrito por SARRUGE (1975).

Quando as plantas estavam com nove meses de idade, determinaram-se a área foliar, os pesos de matéria seca do caule, folhas, raízes e total, assim como a atividade da redutase de nitrato $e$ o teor de nitrogênio total das folhas e raízes.

Os pesos de matéria seca foram obtidos através de secagem do material em estufa a $60^{\circ} \mathrm{C}$ até peso constante. A área foliar foi avaliada a partir do peso de matéria seca de 25 discos de folhas de área conhecida e do peso de matéria seca total das folhas.

A atividade da enzima redutase de nitrato foi determinada pelo método in vivo com e sem adiçāo de nitrato no meio de reaçāo, inicialmente descrito por MEGURO \& MAGALHÄES (1982), e que foi otimizado para as análises em raízes (CARELLI et al., 1990), com borbulhamento contínuo com $\mathrm{N}_{2}$ (para manter a anaerobiose) durante a incubação das amostras de tecido. A atividade da redutase de nitrato foi determinada nas folhas recém-expandidas e nas raizes mais jovens, que são os tecidos que apresentam maior atividade enzimática (CARELLI, 1987). Quatro horas antes das análises enzimáticas, as plantas foram irrigadas com as respectivas soluções nutritivas.

$O$ teor de nitrogênio total foi determinado pelo método de micro-Kjeldahl, após secagem do material em estufa a $60^{\circ} \mathrm{C}$, conforme descrito por BATAGLIA et al. (1983).

Neste experimento, utilizou-se o delineamento estatístico inteiramente casualizado, sendo a análise de crescimento, o teor de nitrogênio total e a atividade enzimática efetuadas, respectivamente, com dez, cinco e quatro repetiçóes para cada tratamento. A comparaçāo entre médias foi feita pelo teste de Tukey a $1 \%$ de probabilidade.

\section{RESULTADOS}

O efeito dos vários niveis de nitrogênio no crescimento das plantas de café é apresentado no quadro 1. Observa-se que o peso de matéria seca das folhas 
aumentou com a concentraçāo de nitrato nas soluçōes nutritivas, enquanto a matéria seca das raizes năo foì alterada. $O$ aumento na produçāo de matéria seca da parte aérea, com o aumento na disponibilidade de nitrogênio para as plantas, foi devido, principalemnte, ao maior peso de matéria seca das folhas, uma vez que apenas a concentração de $15 \mathrm{mM}$ de nitrato proporcionou acréscimo no peso de matéria seca do caule. A relação entre o peso de matéria seca das folhas e das raízes foi de 1,2, 1,8 e 2,2, respectivamente, para as concentraçöes de nitrato de $3,75,7,5$ e $15 \mathrm{mM}$.

QUADRO 1. Efeito de níveis de nitrato na produção de matéria seca em plantas de café de nove meses de idade. Dados médios de dez repetiçóes (')

Nivel de nitrato

Peso de matéria seca

Folha Raiz Caule Parte aérea Total

\begin{tabular}{|c|c|c|c|c|c|}
\hline $\mathrm{mM}$ & & & $\mathrm{g} /$ planta & & \\
\hline $15,00 \ldots \ldots \ldots \ldots \ldots \ldots$ & $4,3 c$ & 2,0 & $1,6 \mathrm{~b}$ & $5,9 \mathrm{c}$ & $7,9 \mathrm{~b}$ \\
\hline $7,50 \ldots \ldots \ldots \ldots \ldots \ldots \ldots$ & $3,0 \mathrm{~b}$ & 1,7 & $1,2 \mathrm{a}$ & $4,2 \mathrm{~b}$ & 5,9 a \\
\hline $3,75 \ldots \ldots \ldots \ldots \ldots \ldots \ldots \ldots \ldots$ & $2,1 \mathrm{a}$ & 1,7 & $1,0 \mathrm{a}$ & $3,1 \mathrm{a}$ & $4,8 \mathrm{a}$ \\
\hline F & $43,57^{\star \star}$ & $1,01 \mathrm{~ns}$ & $16,4^{\star \star}$ & 35,6 ** & $19,2^{\star \star}$ \\
\hline 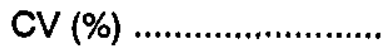 & 17,0 & 23,0 & 20,6 & 17,3 & 14,3 \\
\hline
\end{tabular}

(') Médias seguidas de letras iguais nas colunas náo diferem pelo teste de Tukey $(0,01)$.

No quadro 2, verifica-se que o número de pares de folhas presentes no ramo ortotrófico nāo foi alterado, enquanto a área foliar, do mesmo modo que o peso de matéria seca das folhas, aumentou significativamente com a concentraçāo de nitrato. A altura das plantas aumentou somente com $15 \mathrm{mM}$ de nitrato, de modo análogo ao verificado com o peso de matéria seca do caule.

A atividade da redutase de nitrato analisada sem nitrato, no meio de reação, forma que realmente expressa o que está ocorrendo in situ, foi bem maior nas raizes do que nas folhas - Quadro 3. Verifica-se que a atividade enzimática nas raízes aumentou com a concentração de nitrato no meio de crescimento. Nas folhas, o aumento na atividade da redutase de nitrato foi menos acentuado, náo havendo diferença significativa nas duas maiores concentraçöes de nitrato $(7,5 \mathrm{e}$ $15 \mathrm{mM})$.

A atividade da redutase de nitrato analisada com nitrato no meio de reação, não diferiu nos vários níveis de nitrato, tanto nas folhas como nas raízes 
- Quadro 4. Do mesmo modo que na análise sem nitrato, a atividade enzimática foi maior nas raízes do que nas folhas.

QUADRO 2. Efeito de níveis de nitrato na altura, número de pares de fothas no ramo ortotrópico e na área foliar de plantas de café de nove meses de idade. Média de dez repetiçōes(')

\begin{tabular}{|c|c|c|c|}
\hline Nivel de nitrato & $\begin{array}{c}\text { Altura } \\
\text { da planta }\end{array}$ & $\begin{array}{c}\text { Pares } \\
\text { de folhas }\end{array}$ & $\begin{array}{l}\text { Área } \\
\text { toliar }\end{array}$ \\
\hline 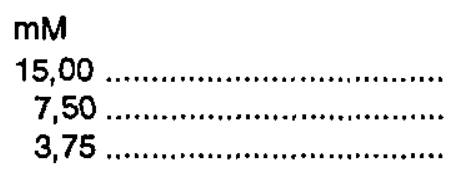 & $\begin{array}{c}\mathrm{cm} \\
26,9 b \\
23,2 a \\
20,9 a\end{array}$ & $\begin{array}{r}n^{2} \\
10 \\
9 \\
10\end{array}$ & $\begin{array}{l}\mathrm{cm}^{2} \\
27,89 \mathrm{c} \\
20,71 \mathrm{~b} \\
13,65 \mathrm{a}\end{array}$ \\
\hline $\mathrm{F}$ CV (\%) & $\begin{array}{l}25,5^{\text {k* }} \\
7,6\end{array}$ & $\begin{array}{l}1,0 \mathrm{~ns} \\
9,3\end{array}$ & $\begin{array}{l}62,15^{\star \star} \\
13,7\end{array}$ \\
\hline
\end{tabular}

(1) Médias seguidas de letras iguais nas colunas, näo diferem pelo teste de Tukey $(0,01)$.

O teor de nitrogênio total nas folhas e nas raízes aumentou somente na concentraçāo de $15 \mathrm{mM}$ de nitrato da solução nutritiva de irrigaçāo - Quadro 3.

QUADRO 3. Atividade da enzima redutase de nitrato, analisada sem nitrato no meio de reaçăo, e teor de nitrogênio total nas folhas e raízes de plantas de café de noves meses de idade, irrigadas com solução nutritiva com três níveis de nitrato. Média de cinco repetiçōes para nitrogênio total e de quatro para atividade enzimática(")

\begin{tabular}{|c|c|c|c|c|}
\hline \multirow{2}{*}{ Nivel de nitrato } & \multicolumn{2}{|c|}{ Redutase de nitrato } & \multicolumn{2}{|c|}{ Nitrogênio total } \\
\hline & Folha & Raiz & Folha & Raiz \\
\hline $\mathrm{mM}$ & \multicolumn{2}{|c|}{$\mu \mathrm{mol} N \mathrm{NO}_{2}^{-} \mathrm{h}^{-1} \mathrm{~g}^{-1} \mathrm{MF}\left({ }^{2}\right)$} & - & - \\
\hline 15,00 & $0,394 b$ & $1,501 \mathrm{c}$ & $3,33 b$ & $3,60 \mathrm{~b}$ \\
\hline $7,50 \ldots \ldots$ & $0,357 \mathrm{~b}$ & $0,942 b$ & $3,07 a$ & $3,17 a$ \\
\hline 3,75 & $0,116 a$ & $0,199 a$ & $2,91 a$ & $3,01 a$ \\
\hline F. & $17,25^{\star \star}$ & $91,57^{\text {t }}$ & $9,00^{\star \star}$ & $33,21^{\text {th }}$ \\
\hline CV (\%) & 20,0 & 13,4 & $5,1 \%$ & 3,62 \\
\hline
\end{tabular}

(1) Médias seguidas de letras iguais nas colunas năo diferem pelo teste de Tukey $(0,01)$.

(7) MF = matéria fresca. 
QUADRO 4. Atividade da redutase de nitrato, analisada com nitrato no meio de reaçäo, nas folhas e raízes de plantas de café de nove meses de idade, irrigadas com solução nutritiva com três níveis de nitrato. Média de quatro repetiçōes.

Nivel de nitrato

Redutase de nitrato

Folha

Raiz

$\mathrm{mM}$

$\mu \mathrm{mol} \mathrm{NO}{ }_{2}^{-1} h^{-1} \mathrm{~g}^{-1} \mathrm{MF}\left(^{2}\right)$

MF(1)

15,00

2,227

3,741

7,50

2,176

3,512

3,75

2,086

3,245

F

$1,06 \mathrm{~ns}$

$1,6 \mathrm{~ns}$

CV $(\%)$

5,64

9,68

(') $\mathrm{MF}$ = matéria fresca.

\section{DISCUSSĀO}

O aumento da disponibilidade de nitrato para as plantas de café proporcionou maior desenvolvimento da parte aérea, principalmente das folhas, em detrimento das raízes, que nāo apresentaram variaçōes. Tal fato já tem sido documentado emespécies anuais (NOVOA\&LOOMIS, 1981; ASLAM \& HUFFAKER, 1982; SUTHERLAND et al., 1985), uma vez que as raizes sāo consideradas como sítios de consumo de assimilados bem menos eficientes do que a parte aérea (CANNELL, 1975; RADIN et al., 1978).

O crescimento e o processo de assimilação de nitrato competem pela energia disponivel nas raízes (RADIN et al., 1978). A atividade da redutase de nitrato nas raízes e o crescimento da parte aérea de plantas de café aumentaram com a concentração de nitrato no meio extemo, enquanto o peso de matéria seca das raizes permaneceu inalterado. Tais resultados sugerem que pode ter ocorrido uma competiçāo pelos carboidratos disponiveis entre o crescimento e a assimilaçăo de nitrato nas raízes.

A distribuiçāo da atividade da redutase de nitrato, entre a raiz e a parte aérea, pode variar com a concentraçăo de nitrato no meio externo e com as condiçōes ambientes (PATE, 1973, 1980). Em espécies anuais, se grandes quantidades de nitrato são fornecidas ao meio radicular, poderá ocorrer uma saturação do sistema de redução de nitrato nas raízes que, consequentemente, exportarāo mais nitrato para as folhas, onde ocorre sua assimilação (WALLACE \& 
PATE, 1965; PATE, 1980; SUTHERLAND et al., 1985). Isso não foi observado em plantas de café, pois os resultados mostraram claramente que o aumento na concentraçāo de nitrato, no meio de crescimento das plantas, aumentou principalmente a atividade enzimática das raízes. Entretanto, deve-se ressaltar que - sistema de redução de nitrato nas raízes nāo estava saturado, uma vez que a atividade da redutase de nitrato nas raizes e, sobretudo, nas folhas, aumentou consideravelmente quando as determinaçōes foram efetuadas com a adiçāo de nitrato exógeno, em relaçāo às análises sem nitrato no meio de reaçāo (Quadros 3 e 4). Portanto, a atividade da redutase de nitrato, tanto nas raízes como nas folhas, foi limitada pela quantidade de substrato. Inicialmente, foi proposto que grande parte das espécies lenhosas reduziriam a maior parte do nitrato absonido em sua raizes (BOLLARD, 1956, 1957; KRAMER \& KOZLOWSKI, 1979; PATE, 1973,1980 ). Entretanto, evidências com base na determinaçăo de alta atividade da redutase de nitrato nas folhas de plantas lenhosas têm sugerido que, em adequados niveis de nitrato, atranslocação e subseqüente reduçāo podem ocorrer eficientemente nesses tecidos (KLEPPER \& HAGEMAN, 1969; ADAMS \& ATTIWILL, 1982; SMIRNOFF et al., 1984; SMIRNOFF \& STEWART, 1985). Os resultados do presente trabalho concordam plenamente com tais consideraçōes.

Os niveis de nitrato fornecidos às plantas pouco alteraram o teor de nitrogênio total nas folhas e nas raízes, indicando que, embora grande quantidade do nitrato tenha sido reduzido nas raizes, parte dos compostos nitrogenados orgânicos foi translocada para as folhas.

\section{CONCLUSÕES}

1) O aumento na disponibilidade de nitrato para as plantas de café proporcionou maior desenvolvimento das folhas, em relação às raízes, cujo crescimento nāo foi alterado.

2) A atividade da enzima redutase de nitrato foi bem maior nas raízes mais jovens do que nas folhas recém-expandidas, diferença essa que se acentuou com o aumento do nitrato disponível às plantas.

\section{REFERÊNCIAS BIBLIOGRÁFICAS}

ADAMS, M.A. \& ATTIWILL, P.M. Nitrate reductase activity and growth response of forest species to ammonium and nitrate sources of nitrogen. Plant and Soil, The Hague, 66(3):373-381, 1982.

ASLAM, M. \& HUFFAKER, R. In vivo nitrate reduction in roots and shoots of barley (Hordeum vulgare L.) seedlings in light and darkness. Plant Physiology, Lancaster, 70(4):1009-1013, 1982. 
BATAGLIA, O.C.; FURLANI, A.M.C.; TEIXEIRA, J.P.F.; FURLANI, P.R. \& GALLO, J.R. Métodos de análise química de plantas. Campinas, Instituto Agronômico, 1983. 48p. (Boletim técnico, 78)

BOLLARD, E.G. Nitrogenous compounds in plant xylem sap. Nature, London, 178(4543):1189-1190, 1956.

Nitrogenous compounds in tracheal sap of woody members of the family Rosaceae. Australian Journal of Biological Sciences, Melbourne, 10(3):288-291, 1957.

CANNELL, M.G.R. Crop physiological aspects of coffee bean yield: a review. Journal of Coffee Research, Karnataka, 5(1/2):7-20, 1975.

CARELLI, M.L.C. Estudo do processo de reduçäo de nitrato durante o desenvolvimento inicial e no estádio reprodutivo de plantas de café (Coffea arabica L.). Campinas, UNICAMP, 1987. 111p. Tese (Doutorado).

; FAHL, J.I. \& MAGALHĀES, A.C. Atividade da redutase de nitrato em folhas e raízes de plantas de café (Coffea arabica L.). Revista Brasileira de Botânica, São Paulo, 13:119-123, 1990.

CHANTAROTWONG, W.; HUFFAKER, R.C.; MILLER, B.L. \& GRANDSTEDT, R.C. In vivo nitrate reduction in relation to nitrate uptake, nitrate content, and in vitronitrate reductase activity in intact barley seedlings. Plant Physiology, Lancaster, 57(4):519-522, 1976.

CRAFTS-BRANDNER, S.J. \& HARPER, J.E. Nitrate reduction by roots of soybean (Glycine max (L.) Merr.) seedlings. Plant Physiology, Lancaster, 69(6):1298-1303, 1982.

HAGEMAN, R.H. \& FLESHER, D. Nitrate reductase activity in corn seedlings as affected by light and nitrate content of nutrient media. Plant Physiology, Lancaster, 35(5):700708, 1960.

HOAGLAND, D.R. \& ARNON, D.I. The water-culture method for growing plants without soil. Berkeley, California Agricultural Experiment Station, 1939. 39. (Circular, 347)

KLEPPER, L. \& HAGEMAN, R.H. The occurrence of nitrate reductase in apple leaves. Plant Physiology, Lancaster, 44(1):110-114, 1969.

KRAMER, P.J. \& KOZLOWSKI, T.T. Nitrogen metabolism and nutrition. In: \& p.302-333. Physiology of wood plants. London, Academic Press, 1979. cap. 9,

LEWIS, O.A.M.;WATSON, E.F. \& HEWITT, E.J. Determination of nitrate reductase activity in barley leaves and roots. Annals of Botany, London, 49(1):31-37, 1982.

MEGURO, N.E. \& MAGALHÄES, A.C. Atividade da redutase de nitrato em cultivares de café. Pesquisa Agropecuária Brasileira, Brasilia, 17(12):1725-1731, 1982.

NOVOA, R. \& LOOMIS, R.S. Nitrogen and plant production. Plant and Soil, The Hague, 58:177-204, 1981. 
PATE, J.S. Transport and partitioning of nitrogenous solutes. Annual Review of Plant Physiology, Palo Alto, 31:313-340, 1980.

. Uptake, assimilation and transport of nitrogen compounds by plants. Soil Biology \& Biochemistry, Oxford, 5(1):109-119, 1973.

QUEIROZ, C.G.S.; ALVES, J.D.; CORDEIRO, A.T. \& RENA, A.B. Distribuiçăo da atividade da redutase de nitrato em Coffea arabica $L$. In: CONGRESSO BRASILEIRO DE PESQUISAS CAFEEIRAS, 12., Caxambu, 1985. Trabalhos apresentados. Rio de Janeiro, IBC/GERCA, 1985. p.137-139.

RADIN, J.W. Contribution of the root system to nitrate assimilation in whole cotton plants. Australian Joumal of Plant Physiology, Melbourne, 4(5):811-819, 1977.

; PARKER, L.L. \& SELL, C.S. Partitioning of sugar between growth and nitrate reduction in cotton roots. Plant Physiology, Lancaster, 62(4):550-553, 1978.

SARRUGE, J.R. Soluçōes nutritivas. SummaPhytopathologica, Piracicaba, 1(3):231-233, 1975.

SCHRADER, L.E. \& THOMAS, R.J. Nitrate uptake, production and transport in the whole plant. In: BEWLEY, J.D., ed. Nitrogen and carbon metabolism. The Hague, Martinus Nijhoff, 1981. cap.3, p. 49-93.

SMIRNOFF, N. \& STEWART, G.R. Nitrate assimilation and translocation by higher plants: comparative physiology and ecological consequences. Physiologia Plantarum, Copenhagen, 64(2):133-140, 1985.

;TODD, P. \& STEWART, G.R. The occurrence of nitrate reduction in the leaves of woody plants. Annals of Botany, London, 54(3):363-374, 1984.

SUTHERLAND, J.M.; ANDREWS, M.; MCINROY, S. \& SPRENT, J.I. The distribution of nitrate assimilation between root and shoot in Vicia fabaL. Annals of Botany, London, 56(2):259-265, 1985.

WALLACE, $W$. The distribution and characteristics of nitrate reductase and glutamate dehydrogenase in the maize seedlings. Plant Physiology, Lancaster, 52(3):191-196, 1973.

\& PATE, J.S. Nitrate reductase in the field pea (Pisum arvense L.). Annals of Botany, London, 29(116):655-671, 1965.

WEISSMAN, G.S. Influence of ammonium and nitrate nutrition on enzimatic activity in soybean and sunflower. Plant Physiology, Lancaster, 49(2):138-141, 1972. 